\title{
INFLUENCE OF THE MICROSTRUCTURE ON CORROSION INDUCED DAMAGE OF WC-Co CEMENTED CARBIDES
}

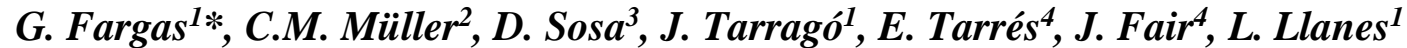 \\ ${ }^{1}$ CIEFMA/EEBE, Departament de Ciència dels Materials i Enginyeria Metal-lúrgica, \\ Universitat Politècnica de Catalunya, Barcelona, Spain \\ ${ }^{2}$ ELECTRODEP, Departament de Ciència dels Materials i Química Física, Universitat de Barcelona, \\ Spain \\ ${ }^{3}$ INTEMA-CONICET, División Metalúrgica, Universidad Nacional de Mar de Plata, Argentina \\ ${ }^{4}$ SANDVIK HYPERION, Coventry, UK \\ e-mail: gemma.fargas@upc.edu
}

\begin{abstract}
The main goal of the present work is to study the influence of the microstructure on the corrosion behavior of cemented carbides WC-Co in two corrosive media: seawater and mine water. Thus, two grades with the same proportion of cobalt as a binder and different grain size: medium and ultrafine were selected. Corrosion kinetics were determined by immersion tests while the electrochemical evolution of the surface was analyzed using impedance testing. Damage tolerance to corrosion was evaluated by assessing fracture strength on specimens previously subjected to corrosion. Results pointed out that for both grades the corrosion rate was higher in sea water, being more significant for the grade with a medium grain size. The corrosion phenomenon that took place in both media was caused by the oxidation reaction of cobalt. In sea water, the polarization resistance decreased for both grades whereas in mine water increased, due to the formation of a layer of corrosion products which slowed down the cobalt dissolution process in surface. In both media, a greater strength loss of the ultrafine grade was evidenced.
\end{abstract}

Keywords: cemented carbides, corrosion kinetics, seawater, mine water

\section{INTRODUCTION}

Cemented carbides are a group of powder metallurgy liquid-phase-sintered materials consisting of brittle refractory carbides embedded in a metallic matrix that acts as a binder. As a consequence of their fully interpenetrated two-phase structure, cemented carbides exhibit an outstanding combination of hardness, wear resistance, strength and toughness. This unique combination of properties has established cemented carbides at the forefront of a wide range of engineering and tooling applications operating under extremely demanding service conditions [1]. However, cemented carbides suffer from different in-service degradation phenomena that seriously affect the performance and service-life of engineering parts. Among them corrosion stands out. Corrosion may develop in a large number of applications where cemented carbides are exposed to chemically aggressive media, including a wide variety of corrosive environments, such as lubricants, chemical and petrochemical products, mine and sea waters $[2,3,4]$. Corrosion damage may induce a detrimental effect on the strength and fatigue resistance of cemented carbides due to the formation of corrosion pits with pronounced stress 
rising effects. Thus, localized corrosion damage may have a key role as a critical flaw, promoting a premature and un-expected failure $[3,4,5,6]$. In the literature, scarce studies have been addressed to correlate corrosion damage and mechanical properties in cemented carbides $[3,4,6]$. In this regard, two most common commercial grades of cemented carbides which present cobalt as a binder were studied. The main goal of the present work was to analyze their corrosion behavior in mine and sea water and to introduce corrosion-induced damage in a controlled way in order to evaluate its effect on fracture strength.

\section{EXPERIMENTAL PROCEDURE}

Two grades of WC-Co, supplied by Sandvik Hyperion, with similar binder content $(15 \%$ wt $)$ and different grain size were studied. In Table 1 main microstructure characteristics: carbide mean grain size $\left(\mathrm{d}_{\mathrm{WC}}\right)$, contiguity $\left(\mathrm{C}_{\mathrm{WC}}\right)$ and binder mean free path $\left(\lambda_{\mathrm{Co}}\right)$ are detailed. A small amount of $\mathrm{Cr}_{3} \mathrm{C}_{2}(0.5 \%$ wt aprox. of binder content $)$ was added to the composition of $15 \mathrm{UF}$ as a grain growth inhibitor as well as increasing corrosion resistance.

Table 1. Nomenclature and microstructural parameters of the studied cemented carbides.

\begin{tabular}{ccccc}
\hline Specimen code & $\begin{array}{c}\text { Binder content } \\
(\text { wt.\%) }\end{array}$ & $\mathbf{d}_{\mathrm{wc}}(\boldsymbol{\mu \mathrm { m }})$ & $\mathbf{C}_{\mathrm{wc}}$ & $\boldsymbol{\lambda}_{\text {binder }}(\boldsymbol{\mu m})$ \\
\hline $15 \mathrm{UF}$ & 15 & $0.5 \pm 0.2$ & $0.4 \pm 0.1$ & $0.2 \pm 0.1$ \\
\hline $15 \mathrm{M}$ & 15 & $1.2 \pm 0.9$ & $0.3 \pm 0.1$ & $0.5 \pm 0.4$ \\
\hline
\end{tabular}

Corrosion kinetics was carried out by immersion tests in stirred synthetic mine water (SMW) and artificial seawater (SW) from 24 to 552 hours. Before immersion tests, specimens were grinded and polished, finalizing with a colloidal silica suspension polishing step. Before and after immersion tests, the specimens were first hand-cleaned by using soapy water and then ultrasonically cleaned for $15 \mathrm{~min}$. in ethanol, subsequently dried with a pure air and weighted in an electronic balance having a resolution of $\pm 0.1 \mathrm{mg}$. Corrosion rates were estimated using the following equation:

$$
\text { Corrosion rate }(\mathrm{mm} / \mathrm{year})=87.6\left(\frac{W}{A \rho T}\right)
$$

where "w" is the weight loss in mg; "A" is the Surface area of the specimen in $\mathrm{cm}^{2}$, " $\rho$ " is the density of the material in $\mathrm{g} / \mathrm{cm}^{3}$; and " $\mathrm{t}$ " is the corrosion time in hours.

Electrochemical tests of polarization resistance and impedance were performed using a Biologic VMP2 potentiostat controlled by Ec-Lab (version 10.38.) software. A standard three-electrodes system was used in which the specimen was the working electrode, a $\mathrm{Nb} / \mathrm{Ti}$ was the counter electrode and $\mathrm{Ag} / \mathrm{AgCl}([\mathrm{NaCl}]=1 \mathrm{M})$ was used as a reference electrode. After immersion in the electrolyte, the open circuit potential was stabilized during $30 \mathrm{~min}$. For polarization resistance measurements, the specimen was polarized into the cathodic region at $-500 \mathrm{MV}$. Then, the potential was increased with a scan rate 
of $600 \mathrm{mV} / \mathrm{h}$ in the positive direction up to $500 \mathrm{mV}$. Electrochemical impedance measurements tests were extended to 340 hours of immersion.

The surface evolution (i.e. corrosion products) after 30, 60, $90 \mathrm{~min}$ and $19 \mathrm{~h}$ was analyzed in detail by atomic force microscopy (AFM) for each grade and studied corrosive media.

After immersion tests, retained flexural strength $\left(\sigma_{\mathrm{r}}\right)$ was assessed by subjecting $4 \mathrm{~mm} \times 3 \mathrm{~mm} \times 45 \mathrm{~mm}$ specimens to failure using a four point bending fully articulated tests with inner and outer spans of 20 and $40 \mathrm{~mm}$ respectively.

\section{RESULTS AND DISCUSSION}

Corrosion rates for the studied cemented carbides grades as a function of immersion time in the two corrosive media are shown in Figure 1. It can be seen that the investigated $15 \mathrm{M}$ grade exhibited much higher corrosion rates than $15 \mathrm{UF}$ grade not only in SMW but also in SW. In this sense and considering the $\lambda_{\text {Co }}$ parameter, medium grain size grade presents a microstructure where the surface of cobalt in contact with the corrosive media is larger and consequently the weight loss is greater too. Differences between SMW and SW in the corrosion rates were more pronounced for the medium grain size grade, $15 \mathrm{M}$, where $\mathrm{SW}$ led to a higher weight loss per unit area in contrast to SMW. For 15UF specimens, these differences were only significant during the first 72 hours of immersion.

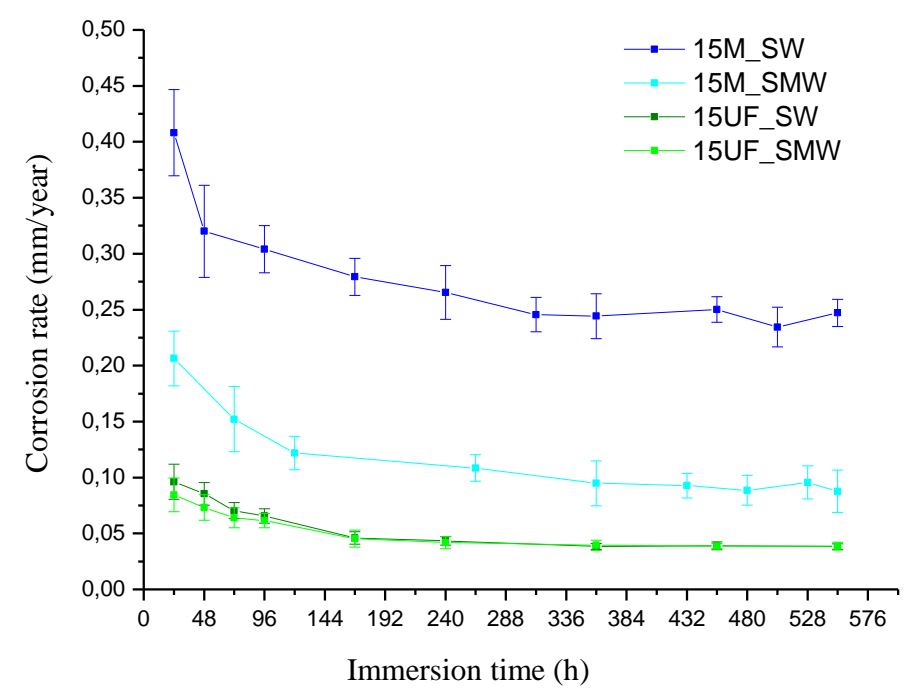

Figure 1. Corrosion rates as a function of immersion time in SMW and SW for the medium and ultrafine grain size studied cemented carbides.

Resulting measures of polarization resistance and impedance pointed out that surface behavior of studied WC-Co grades completely differs in SW compared to SMW. In Figure 2 graphs corresponding to $15 \mathrm{M}$ grade are shown. As it can be observed, values of polarization resistance decreased in SW as increasing immersion time just as the area of the semicircles which became smaller, Figure $2 \mathrm{a}$. These features indicate that the oxidation reaction of cobalt in SW evolves over along dwell time in contact with such 
corrosion medium. Conversely, in SMW, Figure $2 b$, both the polarization resistance and the size of semicircles increased for the first 148 hours. In this case, it is assumed that the dissolution process of cobalt slows down due to some superficial phenomenon related to the formation of corrosion products. From 148 to 340 hours values decreased again as well as the semicircles until displaying similar response of the specimen at the initial state. A similar trend in both corrosion media was observed for 15UF grade.
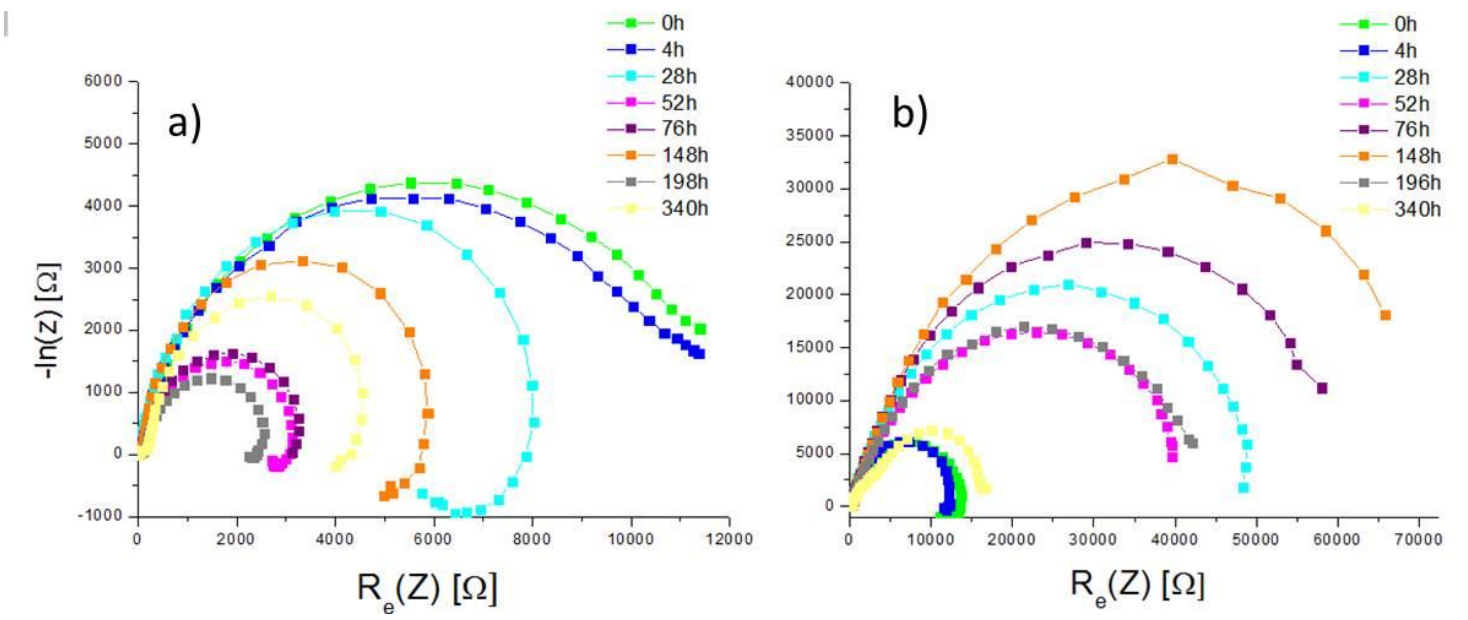

Figure 2. Electrochemical impedance results of 15M grade in: a) SW, b) SMW.

By AFM it was possible to observe that after $30 \mathrm{~min}$ of immersion in SW, Figure 3A, and SMW, Figure 3b, dissolution of cobalt on the surface took place. Differences between both media appeared when comparing samples at larger immersion times (19h). In this case, for both studied grades, specimens immersed in SW showed the formation of corrosion products only in the zone that corresponds to cobalt area, Figure $3 \mathrm{c}$, while in SMW corrosion products cover the entire surface, Figure $3 \mathrm{~d}$. These observations elucidate the results achieved by electrochemical tests regarding the formation of corrosion products and its influence on the difficulty of the cobalt dissolution depending on the corrosive media. 

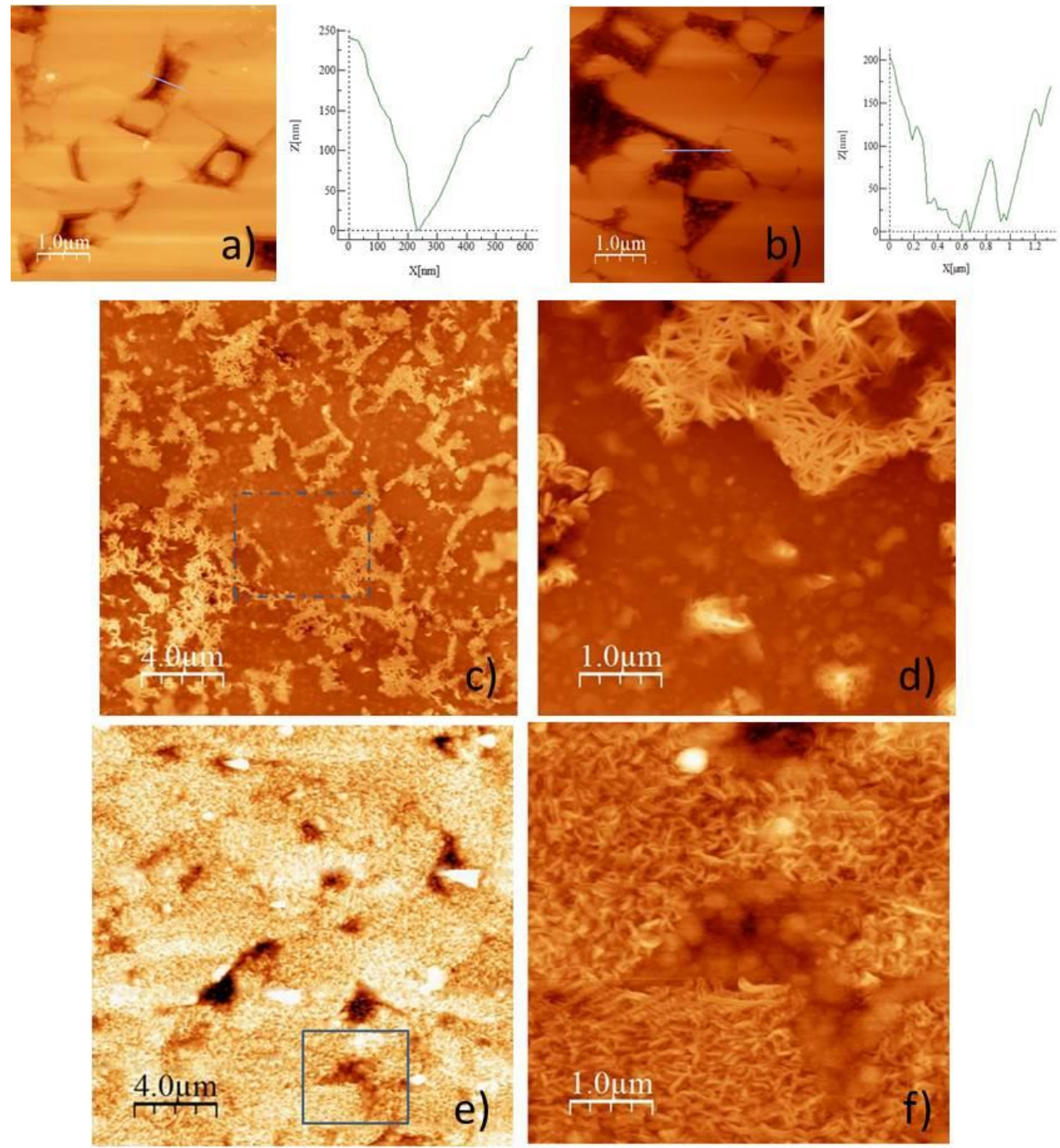

Figure 3. Micrographs of 15M grade obtained by AFM: a) in SW after 30 min. of immersion time, b) in SMW after 30 min. of immersion time, c) in SW after 19h of immersion time, d) magnification of the square in figure c, e) in SMW after 19h of immersion time and f) magnification of the square in figure e.

In Figure 4, normalized strength loss, using as a baseline the strength exhibited by noncorroded specimens, is shown as a function of immersion time. It can be observe that $15 \mathrm{UF}$ grade displayed a greater reduction of fracture strength compared to $15 \mathrm{M}$ grade. This result is in agreement with a previous work [6] in which it was demonstrated that medium grain size microstructure develops uniform corrosion while in ultrafine grain size grades it takes place in terms of pitting corrosion. In this sense, strength loss associated with corrosion damage is rationalized on the basis of stress rising effect related to corrosion pits. Semi-elliptical and sharp angular corrosion pits were identified as critical corrosion damage for medium and ultrafine-sized cemented carbides respectively. The latter have much pronounced stress rising effects and consequently, ultrafine grade exhibits higher strength losses in the presence of corrosion damage. For both grades, specimens immersed in SW displayed fracture strength slightly lower than ones in contact with SMW. 


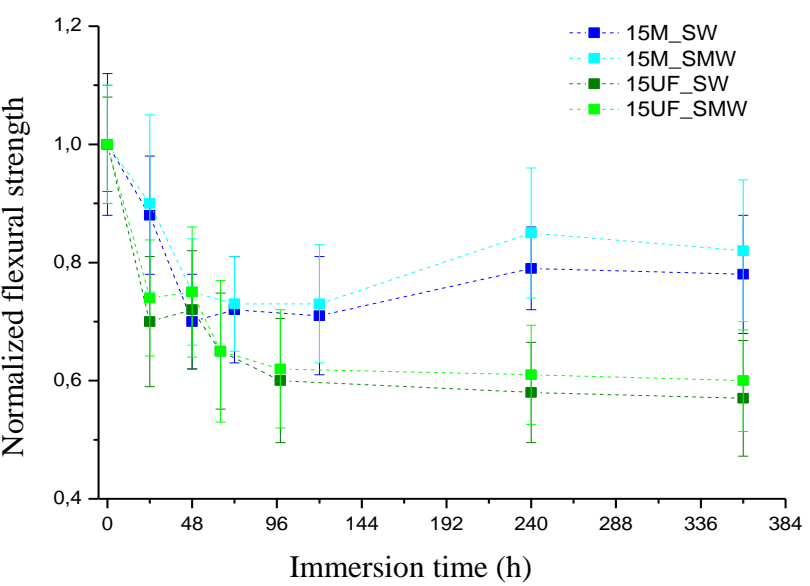

Figure 4. Normalized flexural strength as a function of immersion time for the cemented carbide grades and corrosive media studied

\section{CONCLUSIONES}

The main conclusions resulting from the study can be summarized as follows:

- Medium gran size grain grade displayed higher corrosion rate than ultrafine one for both studied media. In seawater, the corrosion rates were higher than in mine water especially for medium grain size grade.

- Electrochemical tests and AFM observations pointed out the formation of a corrosion products layer in mine water which slows down the oxidation reaction of cobalt. This phenomenon took place at higher degree for the medium grain size grade.

- Corrosion-induced damage led to furthest reduction of fracture strength for ultrafine grain size grade.

\section{REFERENCIAS BIBLIOGRÁFICAS}

[1] L. Prakash, Fundamentals and general applications of hardmetals, in: V.K. Sarin, D. Mari, L. Llanes (Eds.), Comprehensive Hard Materials, Elsevier, UK, 2014: pp. 29-90.

[2] U. Beste, T. Hartzell, H. Engqvist, N. Axén, Surface damage on cemented carbide rock-drill buttons, Wear. 249 (2001) 324-329.

[3] V.A. Pugsley, G. Korn, S. Luyckx, H.G. Sockel, W. Heinrich, M. Wolf, H. Feld, R. Schulte, The influence of a corrosive wood-cutting environment on the mechanical properties of hardmetal tools, Int. J. Refract. Met. Hard Mater. 19 (2001) 311-318.

[4] V.A. Pugsley, H.-G. Sockel, Corrosion fatigue of cemented carbide cutting tool materials, Mater. Sci. Eng. A. 366 (2004) 87-95.

[5] W.J. Tomlinson, I.D. Molyneux, Corrosion, erosion-corrosion, and the flexural strength of WC-Co hardmetals, J. Mater. Sci. 26 (1991) 1605-1608.

[6] J.M. Tarragó, G. Fargas, L. Isern, S. Dorvlo, E.Tarrés, C.M- Müller, E. Jimenez-Piqué, L. Llanes, Microstructural influence on tolerance to corrosion-induced damage in hardmetals, Materials and Design 111 (2016) 36-46. 